\title{
A truncating PET100 variant causing fatal infantile lactic acidosis and isolated cytochrome $c$ oxidase deficiency
}

\author{
Monika Oláhová ${ }^{1}$, Tobias B Haack ${ }^{2,3}$, Charlotte L Alston ${ }^{1}$, Jessica AC Houghton ${ }^{1}$, Langping He ${ }^{1}$, \\ Andrew AM Morris ${ }^{4}$, Garry K Brown ${ }^{5}$, Robert McFarland ${ }^{1}$, Zofia MA Chrzanowska-Lightowlers ${ }^{1}$, \\ Robert N Lightowlers ${ }^{1}$, Holger Prokisch ${ }^{2,3}$ and Robert W Taylor ${ }^{\star, 1}$
}

Isolated mitochondrial complex IV (cytochrome $c$ oxidase) deficiency is an important cause of mitochondrial disease in children and adults. It is genetically heterogeneous, given that both mtDNA-encoded and nuclear-encoded gene products contribute to structural components and assembly factors. Pathogenic variants within these proteins are associated with clinical variability ranging from isolated organ involvement to multisystem disease presentations. Defects in more than 10 complex IV assembly factors have been described including a recent Lebanese founder mutation in PET100 in patients presenting with Leigh syndrome. We report the clinical and molecular investigation of a patient with a fatal, neonatal-onset isolated complex IV deficiency associated with multiorgan involvement born to consanguineous, first-cousin British Asian parents. Exome sequencing revealed a homozygous truncating variant (c.142C $>\mathrm{T}, \mathrm{p} .\left(\mathrm{GIn} 48^{*}\right)$ ) in the PET100 gene that results in a complete loss of enzyme activity and assembly of the holocomplex. Our report confirms PET100 mutation as an important cause of isolated complex IV deficiency outside of the Lebanese population, extending the phenotypic spectrum associated with abnormalities within this gene.

European Journal of Human Genetics (2015) 23, 935-939; doi:10.1038/ejhg.2014.214; published online 8 October 2014

\section{INTRODUCTION}

Mitochondrial oxidative phosphorylation (OXPHOS) is the primary pathway for adenosine triphosphate (ATP) production in eukaryotic cells. This OXPHOS system comprises five transmembrane complexes (I-V) consisting of $\sim 90$ protein subunits that are encoded by either the mitochondria's own genetic material (mtDNA) or the nuclear genome. Of these, complexes I-IV constitute the respiratory chain and complex V, the ATP synthase. Mitochondrial respiratory chain disease is caused by defective OXPHOS and represents a major inborn error of metabolism. ${ }^{1}$ Mitochondrial disease is associated with both a varied age of onset and a diverse spectrum of clinical presentations in which brain, CNS and muscle involvement are common. ${ }^{2}$ The hallmark clinical and genetic heterogeneity of mitochondrial disease is frequently compounded by the lack of clear genotype-phenotype correlations, ${ }^{3}$ although biochemical assessment of respiratory chain complex activities in skeletal muscle is often helpful in guiding molecular genetic diagnostic testing. For many patients, especially children, the genetic aetiology of their condition remains unknown. Complex IV (also known as cytochrome $c$ oxidase (COX)) is the terminal enzyme complex of the mitochondrial respiratory chain, catalysing electron transfer from cytochrome $c$ to molecular oxygen, thus contributing to the proton gradient across the inner mitochondrial membrane that drives ATP synthesis. ${ }^{4}$ The human COX enzyme comprises 14 structural subunits, 3 of which are of mitochondrial origin and form the catalytic core. ${ }^{5,6}$ The remaining components are translated on cytosolic ribosomes and imported into mitochondria. The incorporation of all 14 polypeptides to form a mature complex IV is an intricate process orchestrated by over 20 different assembly factors. ${ }^{5,7}$ Recessively inherited defects in several COX assembly proteins result in the failure to assemble a functional holoenzyme and underlie a number of mitochondrial respiratory chain disease presentations characterised by isolated COX deficiency. The clinical manifestation of COX deficiency includes severe myopathy, cardiomyopathy, liver failure and Leigh syndrome, a progressive, subacute, necrotising encephalopathy that is commonly associated with deleterious variants in the SURF1 gene. ${ }^{8,9}$ SURF1 is an accessory protein related to the yeast $S h y^{10,11}$ that facilitates heme $a$ insertion into COX1 in the early steps of complex IV biogenesis. ${ }^{12,13}$ Although pathogenic variants in a number of other nuclear-encoded complex IV biogenesis factors have been identified (COA5, ${ }^{14}$ TACO $1,{ }^{15}$ LRPPRC, ${ }^{16}$ COX $10,{ }^{17} \mathrm{COX} 15,{ }^{18} \mathrm{SCO} 1,{ }^{19} \mathrm{SCO} 2,{ }^{19}$ and $\mathrm{COX} 20^{20}$ ), the precise mechanism(s) that control COX assembly remain unclear.

Here, we report the application of whole exome sequencing to elucidate the basis of an isolated COX deficiency in a pediatric patient with a severe and fatal neonatal presentation of mitochondrial disease due to a homozygous truncating variant in the PET100 gene. Previous studies in yeast identified PET100 gene as a COX biogenesis factor, ${ }^{21-23}$ and more recently a Lebanese PET100 founder mutation has been described in 10 individuals presenting with Leigh syndrome. ${ }^{24}$ Fibroblasts and skeletal muscle of our patient showed

${ }^{1}$ Wellcome Trust Centre for Mitochondrial Research, Newcastle University, Newcastle upon Tyne, UK; ${ }^{2}$ Institute of Human Genetics, Helmholtz Zentrum München, Neuherberg, Germany; ${ }^{3}$ Institute of Human Genetics, Technische Universität München, Munich, Germany; ${ }^{4}$ Willink Biochemical Genetics Unit, Manchester Centre for Genomic Medicine, Central Manchester University Hospitals NHS Foundation Trust, Manchester, UK; ${ }^{5}$ Department of Biochemistry, University of Oxford, Oxford, UK

${ }^{*}$ Correspondence: Professor RW Taylor, Wellcome Trust Centre for Mitochondrial Research, Institute of Neuroscience, Medical School, Newcastle University, Newcastle upon Tyne NE2 4HH, UK. Tel: +44 191 2083685; Fax: +44 191 2824373; E-mail: robert.taylor@ncl.ac.uk

Received 12 May 2014; revised 23 July 2014; accepted 27 August 2014; published online 8 October 2014 
impaired complex IV activity, associated with a profound defect in COX assembly, and decreased steady-state levels of complex IV proteins. These data provide further evidence that PET100 is an essential factor involved in the maturation and assembly of complex IV.

\section{SUBJECTS AND METHODS}

\section{Patient 1}

Our patient (ID 73387) is a female child, born by an emergency caesarean section at 34 weeks of gestation to consanguineous, first-cousin British Pakistani parents. Antenatal scans showed that she was small for her gestation, weighing $1.19 \mathrm{~kg}$ at birth with a head circumference of $26.7 \mathrm{~cm}$, considerably below the 0.4th centile. Induction of labour had been attempted because of the growth retardation but had failed, leading to the emergency caesarean section. The Apgar scores were 4 at $1 \mathrm{~min}, 7$ at $5 \mathrm{~min}$ and 9 at $10 \mathrm{~min}$. She was admitted to the neonatal intensive care unit for continuous positive airway pressure ventilation.

At a few hours of age, she developed a severe lactic acidosis. The initial lactic acid concentration was $22 \mathrm{mmol} / \mathrm{l}$ and subsequently increased to $63 \mathrm{mmol} / \mathrm{l}$ (normal range, $0.7-2.1 \mathrm{mmol} / \mathrm{l}$ ). She was treated with intravenous infusions of sodium bicarbonate and Tris-hydroxymethyl aminomethane (THAM), but it was never possible to correct the metabolic acidosis. She also developed hypoglycaemia within hours of birth that was corrected with an intravenous infusion of $15 \%$ glucose $(7.8 \mathrm{mg} / \mathrm{kg} / \mathrm{min})$. The ammonia concentration was normal. Urine organic acid profile showed massive excretion of lactic acid and increased phenolic acids, especially hydroxyphenyllactate. Plasma amino acids showed raised concentrations of alanine and glutamine (1567 and $1369 \mu \mathrm{mol} / \mathrm{l}$, respectively), consistent with the lactic acidosis; several other amino acids were also mildly increased. There was gross generalised aminoaciduria. Blood acylcarnitine analysis was normal. Echocardiography showed a structurally normal heart and good ventricular function. Cranial ultrasound showed bilateral intraventricular cysts within the frontal horns and anterior portions of the lateral ventricles. The left-sided cysts were larger, up to $15 \mathrm{~mm}$ in diameter, whereas the largest cyst on the right was $8 \mathrm{~mm}$ in diameter. The choroid plexuses were hyperechoic and irregular, suggesting previous intraventricular haemorrhage. Abdominal ultrasound showed a distended urinary bladder but was otherwise unremarkable. There was severe coagulopathy with an extended prothrombin time of $47.7 \mathrm{~s}$ (normal 12.3-16.6 s), a very low plasma albumin of $7 \mathrm{~g} / \mathrm{l}$ (normal $35-50 \mathrm{~g} / \mathrm{l}$ ), otherwise normal liver function tests but a raised creatine kinase of $2700 \mathrm{U} / 1$ (normal $<300 \mathrm{U} / \mathrm{l}$ ).

She was transferred to a tertiary centre because of her worsening metabolic acidosis. She started having seizures at $\sim 48 \mathrm{~h}$ of age. Despite infusions of bicarbonate and THAM, her acidosis continued to worsen. Muscle and skin biopsies were performed and the family agreed to the withdrawal of intensive care treatment. She died aged $55 \mathrm{~h}$. All documented studies were approved and performed under the ethical guidelines issued by each of our Institutions for clinical studies, with written informed consent obtained from the family.

\section{Cell culture}

Fibroblasts from the affected individual and age-matched controls were cultured in Eagle's minimal essential medium (Sigma, Gillingham, UK) supplemented with $10 \%(\mathrm{v} / \mathrm{v})$ fetal calf serum, $1 \times$ non-essential amino acids, $1 \mathrm{~mm}$ sodium pyruvate and $50 \mu \mathrm{g} / \mathrm{ml}$ uridine, humidified at $37^{\circ} \mathrm{C}$ and $5 \% \mathrm{CO}_{2}$.

\section{Muscle histology and biochemistry}

Informed consents with appropriate ethics review committee approvals were obtained. Histological and histochemical analyses were performed on $10 \mu \mathrm{m}$ transversely orientated serial cryosections of skeletal muscle biopsy samples using standard procedures. The activities of individual respiratory chain complex activities and citrate synthase, a mitochondrial matrix marker, were determined in muscle homogenates and cultured skin fibroblasts as previously described. $^{25}$

\section{Molecular genetics}

Total genomic DNA was obtained using standard methods and the coding region plus intron-exon boundaries of several COX assembly (SURF1, SCO1, SCO2, COX10, COX14, COX15, COA5, LRPPRC, TACO1, FAM37A) an structural (NDUFA4) genes were amplified using locus-specific primers (sequences available upon request), sequenced using the BigDye v3.1 kit and capillary electrophoresed on the ABI3130xl fluorescent sequencing platform (Life Technologies, Warrington, UK).

Whole exome sequencing was undertaken to investigate the genetic basis of this child's mitochondrial disease presentation as previously described. ${ }^{26} \mathrm{~A}$ SureSelect Human All Exon 50 Mb V5 Kit (Agilent, Santa Clara, CA, USA) was used for enrichment of coding DNA fragments and sequencing was performed on a HiSeq2000 system (Illumina, San Diego, CA, USA). BWA (version 0.5.8) was used for read alignment to the human reference assembly (hg19) and single-nucleotide variants (SNVs) and small insertions and deletions were detected with SAMtools (version 0.1 .7 ). The average coverage was 128 -fold and $>97 \%$ of the target region was covered at least 20 -fold allowing for highconfidence variant calls. Detailed sequencing statistics are provided in Table 1.

\section{Cell lyses and western blotting}

Cultured fibroblasts were harvested and lysed in $50 \mathrm{~mm}$ Tris- $\mathrm{HCl} \mathrm{pH} \mathrm{7.5,}$ $130 \mathrm{~mm} \mathrm{NaCl}, 2 \mathrm{~mm} \mathrm{MgCl} 2,1 \mathrm{~mm}$ phenylmethanesulfonyl fluoride (PMSF), $1 \%$ Nonidet P-40 (v/v) and $1 \times$ EDTA free protease inhibitor cocktail (Pierce, Rockford, IL, USA). Protein lysates ( $40 \mu \mathrm{g}$ ) were separated according to size on $12 \%$ gels by sodium dodecyl sulphate-polyacrylamide gel electrophoresis (SDS-PAGE) and electrophoretically transferred to a PVDF membrane (Immobilon-P, Millipore Corporation, Darmstadt, Germany). Immunoblotting was performed using primary and HRP-conjugated secondary antibodies.

\section{Mitochondrial preparation and blue native electrophoresis}

Cultured fibroblasts were harvested, resuspended in homogenisation buffer (HB) $(0.6 \mathrm{~m}$ mannitol, $1 \mathrm{~mm}$ ethylene glycol tetraacetic acid, $10 \mathrm{~mm}$ Tris-HCl $\mathrm{pH} 7.4,1 \mathrm{~mm}$ PMSF and $0.1 \%(\mathrm{v} / \mathrm{v})$ bovine serum albumin (BSA)) and subjected to $3 \times 15$ passes of homogenisation using a Teflon glass Dounce homogeniser at $4{ }^{\circ} \mathrm{C}$. Standard differential centrifugation ( $400 \mathrm{~g}$ for $10 \mathrm{~min}$ ) was used to remove nuclei and cell debris and mitochondria were finally pelleted at $11000 \mathrm{~g}$ for $10 \mathrm{~min}$ at $4{ }^{\circ} \mathrm{C}$. Mitochondria were washed in HB without BSA and the final pellet was solubilised by $n$-Dodecyl $\beta$-D-maltoside (DDM) (Sigma) at $2 \mathrm{mg} / \mathrm{mg}$ protein on ice for $20 \mathrm{~min}$. Following centrifugation $(100000 \mathrm{~g}$ for 15 min at $4{ }^{\circ} \mathrm{C}$ ) the supernatant was collected and Coomassie Blue G-250 (AMS Biotechnology (Europe) Ltd, Abingdon, UK) was added. Mitochondria membrane proteins $(50 \mu \mathrm{g})$ were loaded on a NativePAGE $4-16 \%$ BisTris gel (Life Technologies), electrophoretically separated and transferred to a PVDF membrane. The membrane was subsequently immunoblotted with antibodies raised against OXPHOS complexes.

\section{Immunoblotting}

The following primary antibodies were used for immunoblotting: NDUFA9 (Molecular Probes, Eugene, OR, USA, A21344), NDUFB8 (Abcam, Cambridge, UK, ab110242), SDHA (MitoSciences, Eugene, OR, USA, MS204), UQCRC2 (Abcam, ab14745), COX1 (Abcam, ab14705) and COX2 (Molecular Probes, A6404), ATP5A (Abcam, ab14748), ATPB (Abcam, ab14730) and TOM20

\section{Table 1 Variants identified at different filtering levels in individual no. 73387}

\section{Variants filtering}

Synonymous variants 11836

Nonsynonymous variants (NSVs)

NSVs absent from 3600 control exomes and public databases

Genes carrying $\geq 2$ NSV 
(Santa Cruz, Heidelberg, Germany, sc11415). HRP-conjugated anti-mouse or anti-rabbit secondary antibodies were used (P0260 and P0399 respectively; Dako, Glostrup, Denmark). Chemiluminescence ECL Prime Kit (Amersham, Little Chalfont, UK) and ChemiDocMP Imaging System (Bio-Rad, Hemel Hempstead, UK) were used for signal detection and Image lab 4.0.1 (Bio-Rad) software for analysis.

\section{RESULTS}

Muscle histochemistry and respiratory chain analyses

Analysis of the patient's muscle biopsy demonstrated a severe and global loss of COX histochemical activity throughout the section (not shown), confirmed by the spectrophotometric assay of respiratory chain activities, that demonstrated a severe and isolated deficiency of complex IV in muscle homogenates (Figure 1a). This observation was confirmed in patient fibroblasts in which complex IV activity was markedly decreased (Figure $1 \mathrm{~b}$ ).

\section{Molecular genetic studies identify a novel truncating PET100 variant}

Sanger sequencing of several COX assembly genes and structural components did not identify causative variants, prompting whole exome sequencing. Prioritisation of candidate disease genes was performed essentially as reported previously, ${ }^{27}$ with our analysis focussing on nonsynonymous variants. Based on the rare disease phenotype we expected disease-causal variants to have a low frequency in the general population, and hence we excluded detected variants present in 3600 control exomes and public databases. Assuming an autosomal recessive mode of inheritance, we searched for genes carrying predicted compound heterozygous or homozygous variants (Table 1). This filter left 38 genes. Two genes, namely MYH14 $\left(\mathrm{MIM}^{\star} 608568\right)$ and ANKS6 $\left(\mathrm{MIM}^{\star} 615370\right)$, have been previously

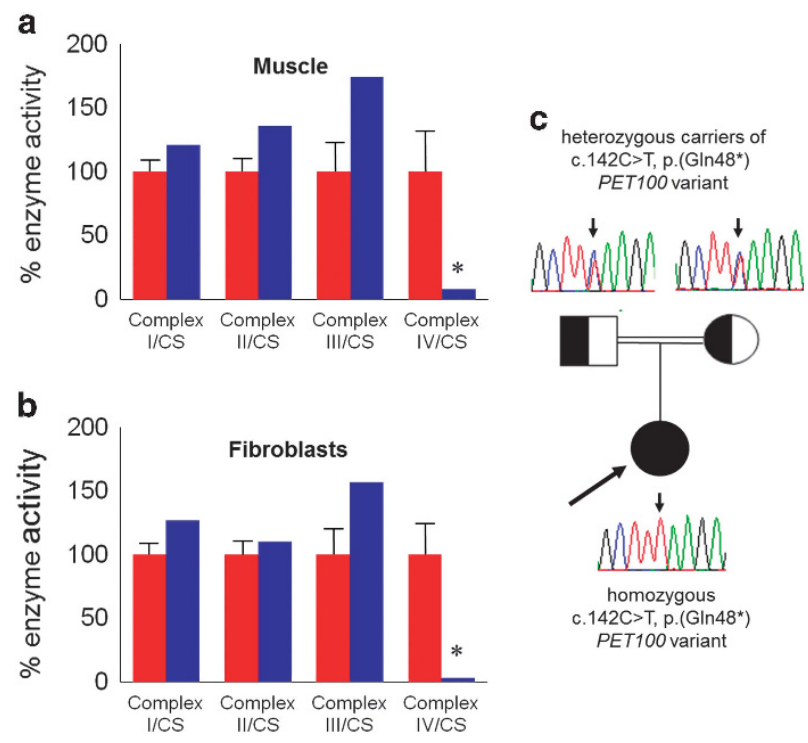

Figure 1 Identification of an isolated mitochondrial respiratory chain complex IV deficiency in muscle and fibroblasts and analysis of PET100 variant. The assessment of individual respiratory chain enzyme activities in muscle (a) and fibroblasts (b) identified a severe OXPHOS deficiency affecting complex IV in isolation in the patient (blue bars) compared with controls (red bars); mean enzyme activities shown for muscle controls ( $n=25)$ and fibroblast controls $(n=10)$ are set at $100 \%$. (c) Family pedigree showing confirmation of p. $\left(\mathrm{Gln} 48^{*}\right)$ carrier status in clinically unaffected parents, whereas the proband is homozygous for the truncating variant. linked to human disease. Variants in both were excluded as likely candidates because of different clinical presentations, reportedly autosomal dominant mode of inheritance in the case of MYH14, and the fact that both heterozygous ANKS6 variants were confirmed to be in cis on the same allele. Only one gene, PET100 (NM_001171155.1), carried two predicted loss-of-function alleles. The patient was homozygous, for a truncating PET100 variant (c. $[142 \mathrm{C}>\mathrm{T}] ;[142 \mathrm{C}>\mathrm{T}]$, p. $\left[\left(\mathrm{G} \ln 48^{\star}\right)\right] ;\left[\left(\mathrm{G} \ln 48^{*}\right)\right]$; ClinVar Reference ID: mdi-3317) that resides in the fourth coding exon and predicts a truncated protein in which the last 26 amino acids are lost (33\% of the full-length protein). Concordant with a disease-causal role of the homozygous (c.142C > T, p. $\left.\left(\mathrm{Gln} 48^{*}\right)\right)$ variant, confirmatory Sanger sequencing revealed that both healthy parents were heterozygous carriers (Figure 1c).

\section{Mutation of PET100 leads to impaired complex IV assembly}

Further characterisation of the nature of the biochemical defect associated with the PET100 variant was performed in patient fibroblasts. The steady-state levels of individual OXPHOS complex subunits and the subsequent assembly into mitochondrial respiratory chain complexes were analyzed by Blue-native PAGE (BN-PAGE) and SDS-PAGE respectively.

The analysis of the steady-state levels of OXPHOS complex components confirmed a marked decrease in COXI and COXII in patient compared with control fibroblasts (Figure 2a). No significant changes were detected in any of the steady-state levels of all other analyzed complexes (I, II, III and V), although in agreement with the respiratory chain enzyme results in muscle, there was a suggestion that complex III levels were increased $\sim 1$.6-fold based on densitometric analysis (Figure 2a). A similar observation has been made previously in patients harbouring pathogenic variants in another COX assembly gene, SURF1. ${ }^{28}$ TOM20 was used as a mitochondrial loading marker and confirmed equal loading of control and patient mitochondrial protein.

Consistent with this reduction in steady-state levels of CIV proteins and the biochemical measurements of the respiratory chain complex activities (Figure 1b), BN-PAGE analysis revealed significantly decreased amounts of fully assembled complex IV in patient cells compared with age-matched controls (Figure 2b). This loss of OXPHOS complex was specific as the assembly profile of complexes I, II, III and V were normal.

These data demonstrate that the consequence of the homozygous $\mathrm{p}$. $\left(\mathrm{Gln} 48^{\star}\right)$ PET100 variant is a specific and severe loss of COX subunits and fully assembled complex IV.

\section{DISCUSSION}

Although recognised as one of the most common energy metabolism disorders, isolated COX deficiency has a diverse genetic aetiology that reflects the complex nature of biogenesis and assembly of mtDNAand nuclear-encoded components into mature holoenzyme; a process facilitated by numerous chaperone proteins. Pathogenic variants in a number of the assembly factors necessary for the formation of a functional COX enzyme have been reported. ${ }^{9,14-20}$ Recently, a founder mutation in a highly conserved COX assembly factor PET100 has been identified in 10 Lebanese individuals with isolated COX deficiency who present with Leigh syndrome and seizures. ${ }^{24}$

Here we report that a new truncating PET100 variant causes fatal infantile lactic acidosis and isolated COX deficiency in a child born to consanguineous British Pakistani parents. The pathogenic nonsense (c. $142 \mathrm{C}>\mathrm{T}, \mathrm{p} .\left(\mathrm{G} \ln 48^{\star}\right)$ ) variant in the PET100 gene was identified by whole exome sequencing, leading to impaired complex IV enzyme 


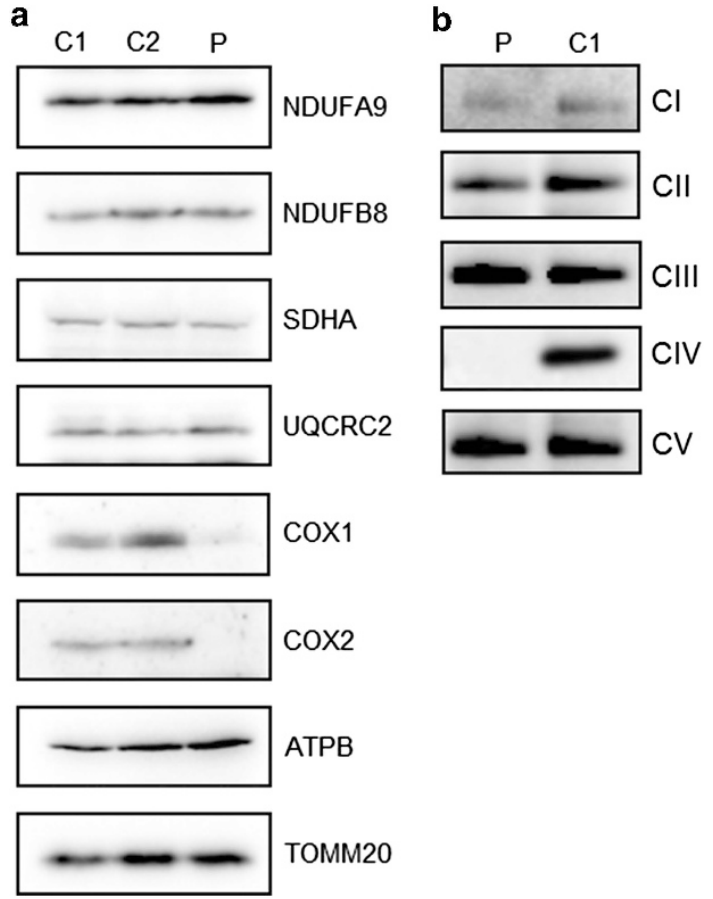

Figure 2 Steady-state levels of OXPHOS components and complexes. (a) Cell lysate from control ( $\mathrm{C} 1$ and $\mathrm{C} 2)$ and patient $(\mathrm{P})$ fibroblasts $(40 \mu \mathrm{g})$ were analysed by SDS-PAGE (12\%) and immunoblotting. Subunit-specific antibodies were used against $\mathrm{Cl}$ (NDUFA9, NDUFB8), CII (SDHA), CIII (UQCRC2), CIV (COX1, COX2) and CV (ATPB). The outer mitochondrial membrane marker, TOM20, was used as a loading control. (b) Mitochondrial proteins $(50 \mu \mathrm{g})$ isolated from patient (P) and control (C1) fibroblasts were analysed by one-dimensional BN-PAGE (4 to $16 \%$ gradient) using subunitspecific antibodies as indicated (CI (NDUFA9), CII (SDHA), CIII (UQCRC2), CIV (COX1) and CV (ATP5A)) to assess the assembly of individual OXPHOS complexes. Complex II (SDHA) was used as a loading control.

activity and abnormal COX assembly. Our results are consistent with previously published data suggesting that PET100 is a conserved biogenesis factor involved in the maturation of complex IV in both humans ${ }^{24}$ and yeast. ${ }^{21-23}$ The yeast homologue of PET100 is not necessary for the localisation of COX polypeptides to the inner mitochondrial membrane, ${ }^{21}$ but it has a major role in the later assembly processes where it facilitates the assembly of COX intermediates. ${ }^{23}$ In contrast, human PET100 appears to be required earlier in the process for the assembly of mitochondrial-encoded COX subunits. ${ }^{24}$ Our results demonstrate the importance of PET100 in OXPHOS function and support previous studies, ${ }^{21-24}$ however, it requires further investigation to fully understand the exact role of this enzyme in the maturation of the COX holoenzyme.

The complex IV assembly profile observed in our patient with this truncating PET100 variant is similar to the reported Lebanese (c.3G $>$ C, p.?) PET100 variant that eliminates the initiation codon potentially resulting in a nonfunctional protein. ${ }^{24}$ However, our study has identified some key differences in the biochemical and clinical disease presentations between the two variants. The residual complex IV enzyme activities were lower in our patient's fibroblasts and skeletal muscle compared with the residual COX activities demonstrated in tissues from the Lebanese patients. The COX defect in the patients carrying the Lebanese (c.3G > C, p.?) variant was associated with Leigh syndrome, seizures, developmental delay and elevated blood lactate levels, although these were variable (ranging from normal to $11 \mathrm{mmol} / \mathrm{l}$ ). ${ }^{24}$ These symptoms were apparent a few months after birth. In contrast, the onset of the disease in our patient was before birth and her lactate levels were extremely high $(63 \mathrm{mmol} / \mathrm{l}$ at its peak). Further differences in our patient's clinical presentation were marked hypoglycaemia, severely impaired liver function and raised creatine kinase reflecting profound disruption of metabolic energy homeostasis. These observations in our patient suggest that impairment of PET100 can lead to severe complications, including prenatal onset and neonatal death, not observed in the other reported PET100 variant. Interestingly, Lebanese individuals harbouring the PET100 truncating variant differ from patients with mutations in SURF1, a different COX assembly factor, in that seizures appear to have an earlier age of onset. ${ }^{24,29}$ Consistent with this, our microcephalic patient showed abnormalities on neuroimaging and suffered seizures from $48 \mathrm{~h}$ of age that are likely to reflect severe problems with in utero brain development. The truncating nature of the PET100 variant may cause the protein to be subject to nonsense-mediated mRNA decay or may otherwise exert a dominant negative effect that in turn determines the severity and early appearance of clinical disease. Importantly, although a PET100 variant has only been identified in patients originating from Lebanon to date, our patient shows that mutations within this gene occur outside of this particular ethnic group.

Whole exome sequencing is a rapid and effective approach to elucidate the molecular bases of mitochondrial respiratory chain disorders including isolated COX deficiency. ${ }^{30,31}$ Our findings confirm PET100 as an important candidate disease gene in patients with isolated COX deficiency. Recent advances in next-generation sequencing enable the rapid and accurate diagnosis of singleton mitochondrial disease patients within small families, thus facilitating appropriate counselling and the offer of preventive strategies, such as prenatal diagnosis and preimplantation genetic profiling.

\section{CONFLICT OF INTEREST}

The authors declare no conflict of interest.

\section{ACKNOWLEDGEMENTS}

This work was supported by a Wellcome Trust Strategic Award (096919/Z/11/Z; to ZMAC-L, RNL and RWT), the MRC Centre for Neuromuscular Diseases (G0601943; to RM and RWT), the Lily Foundation (to RM and RWT), the UK NHS Highly Specialised 'Rare Mitochondrial Disorders of Adults and Children' Service in Newcastle upon Tyne, the BMBF-funded German Network for Mitochondrial Disorders (mitoNET no. 01GM1113C/D), an NIHR/CSO Healthcare Science Research Fellowship from the National Institute for Health Research (NIHR-HCS-D12-03-04; to CLA) and by E-Rare project GENOMIT (01GM1207) funding to HP. We thank S Loesecke and S Schäfer for excellent technical support.

\footnotetext{
1 Schaefer AM, McFarland R, Blakely EL et al: Prevalence of mitochondrial DNA disease in adults. Ann Neurol 2008; 63: 35-39.

2 McFarland R, Taylor RW, Turnbull DM: A neurological perspective on mitochondrial disease. Lancet Neurol 2010; 9: 829-840.

3 Spiegel R, Mandel H, Saada A et al: Delineation of C12orf65-related phenotypes: a genotype-phenotype relationship. Eur J Hum Genet 2014; 22: 1019-1025.

4 Belevich I, Verkhovsky MI, Wikström M: Proton-coupled electron transfer drives the proton pump of cytochrome $c$ oxidase. Nature 2006; 440: 829-832.

5 Mick DU, Fox TD, Rehling P: Inventory control: cytochrome $c$ oxidase assembly regulates mitochondrial translation. Nat Rev Mol Cell Biol 2011; 12: 14-20.

6 Balsa E, Marco R, Perales-Clemente E et al: NDUFA4 is a subunit of complex IV of the mammalian electron transport chain. Cell Metab 2012; 16: 378-386.

7 Ghezzi D, Zeviani M: Assembly factors of human mitochondrial respiratory chain complexes: physiology and pathophysiology. Adv Exp Med Biol 2012; 748: 65-106.

8 Leigh D: Subacute necrotizing encephalomyelopathy in an infant. J Neurol Neurosurg Psychiatry 1951; 14: 216-221.

9 Zhu Z, Yao J, Johns T et al: SURF1, encoding a factor involved in the biogenesis of cytochrome $c$ oxidase, is mutated in Leigh syndrome. Nat Genet 1998; 20: 337-343.
} 
10 Tiranti V, Hoertnagel K, Carrozzo R et al: Mutations of SURF-1 in Leigh disease associated with cytochrome $c$ oxidase deficiency. Am J Hum Genet 1998; 63: 1609-1621.

11 Tiranti V, Galimberti C, Nijtmans L, Bovolenta S, Perini MP, Zeviani M: Characterization of SURF-1 expression and Surf-1p function in normal and disease conditions. Hum $\mathrm{Mol}$ Genet 1999; 8: 2533-2540.

12 Smith D, Gray J, Mitchell L, Antholine WE, Hosler JP: Assembly of cytochrome-c oxidase in the absence of assembly protein Surf1p leads to loss of the active site heme. J Biol Chem 2005; 280: 17652-17656.

13 Bundschuh FA, Hannappel A, Anderka O, Ludwig B: Surf1, associated with Leigh syndrome in humans, is a heme-binding protein in bacterial oxidase biogenesis. $J$ Biol Chem 2009; 284: 25735-25741.

14 Huigsloot M, Nijtmans LG, Szklarczyk R et al: A mutation in C2orf64 causes impaired cytochrome $c$ oxidase assembly and mitochondrial cardiomyopathy. Am J Hum Genet 2011; 88: 488-493.

15 Weraarpachai W, Antonicka H, Sasarman F et al: Mutation in TACO1, encoding a translational activator of $\mathrm{COX} \mathrm{I}$, results in cytochrome $c$ oxidase deficiency and late-onset Leigh syndrome. Nat Genet 2009; 41: 833-837.

$16 \mathrm{Xu} \mathrm{F}$, Morin C, Mitchell G, Ackerley C, Robinson BH: The role of the LRPPRC (leucine-rich pentatricopeptide repeat cassette) gene in cytochrome oxidase assembly: mutation causes lowered levels of COX (cytochrome $c$ oxidase) I and COX III mRNA. Biochem J 2004; 382: 331-336.

17 Antonicka H, Leary SC, Guercin GH et al: Mutations in COX10 result in a defect in mitochondrial heme A biosynthesis and account for multiple, early-onset clinical phenotypes associated with isolated COX deficiency. Hum Mol Genet 2003; 12 : 2693-2702.

18 Bareth B, Dennerlein S, Mick DU, Nikolov M, Urlaub H, Rehling P: The heme a synthase Cox 15 associates with cytochrome $c$ oxidase assembly intermediates during Cox1 maturation. Mol Cel Biol 2013; 33: 4128-4137.

19 Leary SC, Kaufman BA, Pellecchia G et al: Human SCO1 and SCO2 have independent, cooperative functions in copper delivery to cytochrome $c$ oxidase. Hum $\mathrm{Mol}$ Genet 2004; 13: 1839-1848.

20 Bourens M, Boulet A, Leary SC, Barrientos A: Human COX20 cooperates with SCO1 and $\mathrm{SCO} 2$ to mature $\mathrm{COX} 2$ and promote the assembly of cytochrome $c$ oxidase. Hum Mol Genet 2014; 23: 2901-2913.

21 Church C, Chapon C, Poyton RO: Cloning and characterization of PET100, a gene required for the assembly of yeast cytochrome $c$ oxidase. J Biol Chem 1996; 271: 18499-18507.
22 Forsha D, Church C, Wazny P, Poyton RO: Structure and function of Pet100p, a molecular chaperone required for the assembly of cytochrome $c$ oxidase in Saccharomyces cerevisiae. Biochem Soc Trans 2001; 29: 436-441.

23 Church C, Goehring B, Forsha D, Wazny P, Poyton RO: A role for Pet100p in the assembly of yeast cytochrome $c$ oxidase: interaction with a subassembly that accumulates in a pet100 mutant. J Biol Chem 2005; 280: 1854-1863.

$24 \mathrm{Lim}$ SC, Smith KR, Stroud DA et al: Founder mutation in PET100 causes isolated complex IV deficiency in Lebanese individuals with Leigh syndrome. Am J Hum Genet 2014; 94: 209-222.

25 Kirby DM, Thorburn DR, Turnbull DM, Taylor RW: Biochemical assays of respiratory chain complex activity. Methods Cell Biol 2007; 80: 93-119.

26 Mayr JA, Haack TB, Graf E et al: Lack of the mitochondrial protein acylglycerol kinase causes Sengers syndrome. Am J Hum Genet 2012; 90: 314-320.

27 Haack TB, Haberberger B, Frisch EM et al: Molecular diagnosis in mitochondrial complex I deficiency using exome sequencing. J Med Genet 2012; 49: 277-283.

28 Kovářová $N$, Čížková Vrbacká $A$, Pecina $P$ et al: Adaptation of respiratory chain biogenesis to cytochrome $c$ oxidase deficiency caused by SURF1 gene mutations. Biochim Biophys Acta 2012; 1822: 1114-1124.

29 Wedatilake Y, Brown R, McFarland R et al: SURF1 deficiency: a multi-centre natural history study. Orphanet J Rare Dis 2013; 8: 96.

30 DaRe JT, Vasta V, Penn J, Tran NT, Hahn SH: Targeted exome sequencing for mitochondrial disorders reveals high genetic heterogeneity. BMC Med Genet 2013; 14: 118.

31 McCormick E, Place E, Falk MJ: Molecular genetic testing for mitochondrial disease: from one generation to the next. Neurotherapeutics 2013; 10: 251-261.

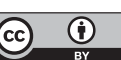

This work is licensed under a Creative Commons Attribution 3.0 Unported License. The images or other third party material in this article are included in the article's Creative Commons license, unless indicated otherwise in the credit line; if the material is not included under the Creative Commons license, users will need to obtain permission from the license holder to reproduce the material. To view a copy of this license, visit http:// creativecommons.org/licenses/by/3.0/ 